\title{
What We Wore and Sketchfab
}

\author{
Rob Gallagher
}

King's College London

\section{WHAT WE WORE}

http://what-we-wore.com/

Maintained by photographer and curator Nina Manandhar, What We Wore describes itself as 'a people's style history of Britain'. I first encountered it on Tumblr, but in the past five years the project has spread to other social media platforms, spawned a coffee table book, exhibitions and workshops and given rise to spin-off devoted to the sartorial history of London's seedily bohemian Soho. In a sense, its appeal is straightforward: other people's photo albums always contain marvels, and the stories and images collected here are often fantastically evocative. The goal of compiling a 'people's style history' is, however, a complicated one, the democratic valences of 'people' rubbing up against the fact that 'style' works to set us apart even as it fosters a sense of belonging. Placing contributions from well-connected players in the music and fashion industries alongside those of everyday folk, the archive testifies to the complex relationship between the popular and the avant-garde in UK culture, while contributors' stories often bear witness to ethnic and socioeconomic divisions. As with other crowdsourced life writing projects that have moved from the screen to page (and as with social media in general), WWW also raises questions as to where providing a platform shades into capitalizing on others' creativity. One could accuse it of pandering to nostalgia too-though, from the 'post-subcultural' perspective of the $21^{\text {st }}$ century, the fidelity to particular genres, looks and scenes on display here is as bracing as it is quaint. Getting a tattoo wasn't always compatible with getting a job, after all.

In Camera Lucida, Barthes famously distinguished between photographic details that belong to the domain of the 'studium' (what the photo represents, what we can infer or interpret from it) and those which possess the penetrating force of the 'punctum' (viscerally affecting 


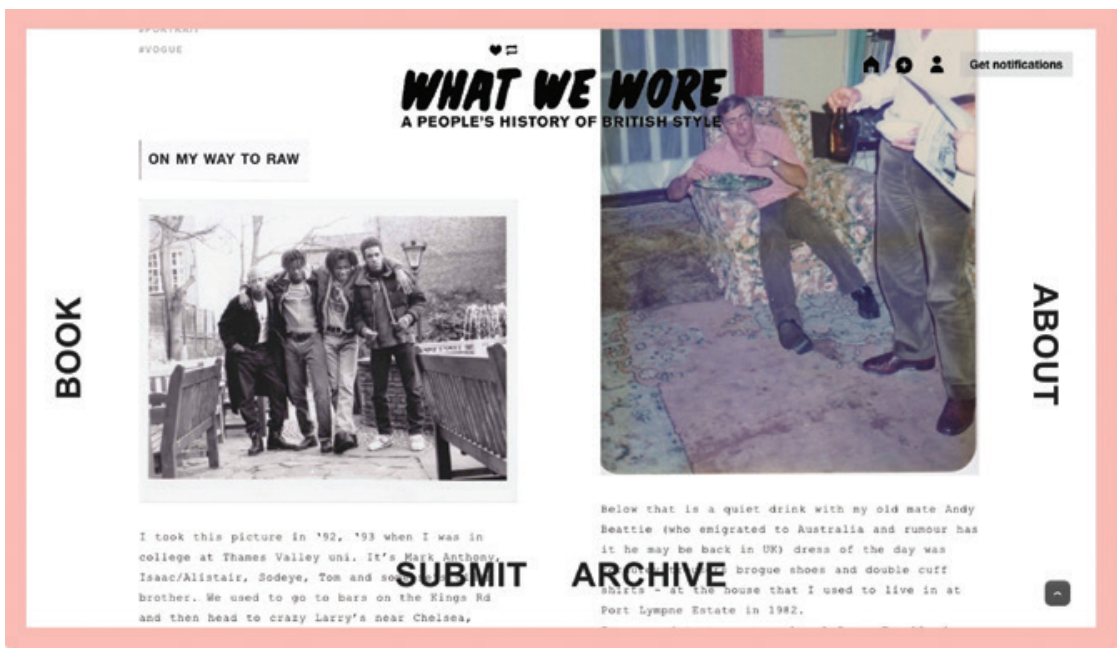

contingencies to which it proves impossible to assign a meaning). As the creation, classification and circulation of images becomes a joint human-algorithmic effort, our understanding of these concepts necessarily changes. Hashtags, metadata, search engines and neural networks become elements of a cybernetic studium, while the force of the punctum can be quantified in terms of how many users feel compelled to momentarily arrest the endless scroll of their Tumblr feeds and tap 'like'. Browsing WWW, I'm always intrigued to see which aspects of a photo or an outfit are considered worthy of a mention in the tags or captions. In a 2013 post feminist designer Karen Savage cringes at a photo of herself in an 'awful' fleece that, in 2018, is again the height of fashion, suggesting how stylistic choices that might strike us as naff or gauche today (if they strike us at all) may attain a new salience-and saleability-tomorrow. Beyond the obvious 'period details' (70s flares, 90s sportswear brands) it's often incidental things that most powerfully evoke the past. In one of the WhatSohoWore images posted to Instagram, stylist Simon Foxton poses in what is apparently a Central Saint Martin's classroom, hip thrust forward to show off the red and white Walkman (then cutting edge, now boxy and primitive-looking) clipped to his belt. Very striking, to be sure, and eminently, deliciously of its time-particularly in combination with the oatmeal suit and blancmange-coloured V-neck. What dates the image for me, though, is the wire mesh safety glass behind Foxton, once omnipresent in institutional buildings, but since (Wikipedia tells me) banned in many countries, having proven less safe than supposed-another casualty of history. 


\section{SKETCHFAB}

https://sketchfab.com/moving_past_present

The link above leads to a profile page on Sketchfab, a database of 3D models and animation files. The files hosted there are the result of a collaboration between artist Janina Lange and King's College London's Strandlines project. Based on the Centre for Life-Writing Research, Strandlines is devoted to documenting 'life on the Strand, past, present and creative'. In 2016 we invited Janina to digitally 'reanimate' Edwardian musical comedy stars Constance Collier and Ellaline Terriss, erstwhile stars of the Strand's Gaiety theatre. Working with CG animator and engineer Moses Attah, Janina created a pop-up motion capture studio, where she recorded performer Meghan Treadway recreating snatches of Collier and Terriss' screen performances-from comic dances to melodramatic death scenes-using an Xbox Kinect device.

The Kinect 'sees' the human body as a simplified skeleton with 26 points of articulation, and can track this geometric model's position in Euclidean space in real time. Separating Meghan's movements (which were themselves recreations of Collier's and Terriss' movements, as captured on celluloid) from her likeness, the Kinect abstracted her live performance into a series of spatiotemporal scripts that can be downloaded for other digital bodies to follow.

On Sketchfab these movements are performed by an avatar resembling a crash test dummy. Janina had initially planned to use one of the default

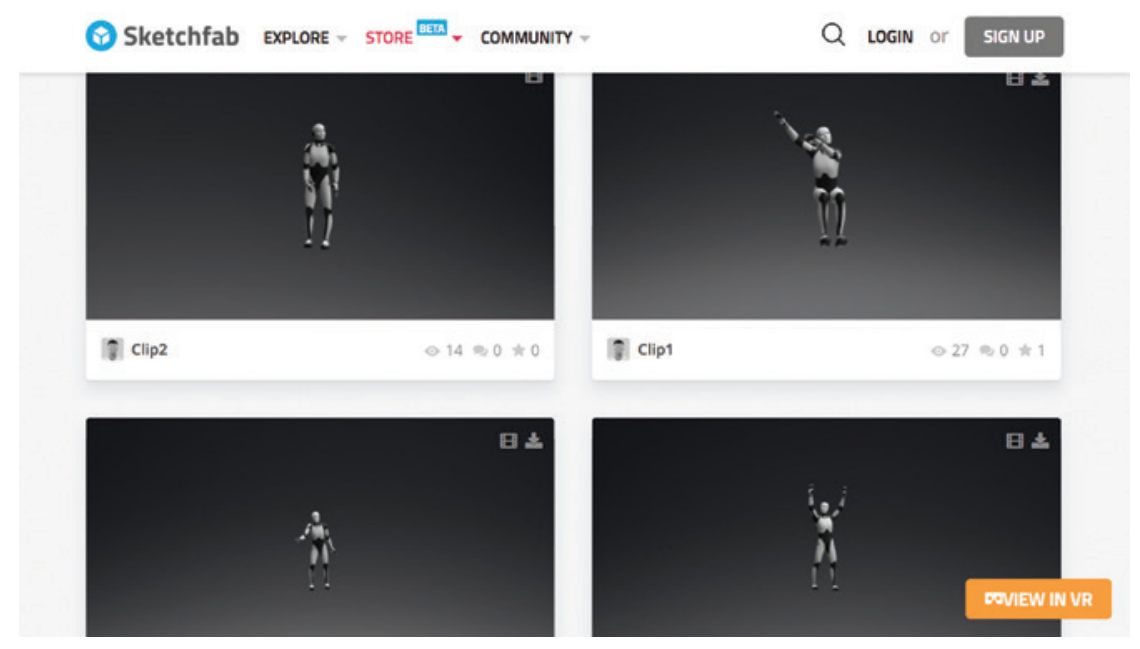


avatars available in the Unreal engine, a popular videogame development tool. After encountering problems with getting Unreal to talk to the modeling and animation program Maya, however, she and Moses were forced to create a bespoke virtual body using Autodesk's Character Generator software. Where many off-the-peg avatars are hyperbolically gendered ('female' bodies tend to be improbably curvaceous, 'male' ones ruggedly trapezoid), Janina wanted to create something more androgynous. By selecting from the libraries of body parts available in Autodesk's libraries and tweaking the model's face, she and Moses arrived at what you see on Sketchfab.

The performances this dummy gives are, in gamer parlance, a little 'janky'-feet bend in ways that feet aren't supposed to bend, limbs hiccup or jitter in a manner Meghan's certainly didn't. As with the syncopated stuttering familiar to viewers of early film, these errata bear witness to the limitations of nascent capture technologies. Those technologies are, however, improving quickly. If smartphone users are already becoming familiar with voice and face recognition systems, in many contexts it is far easier and more practical to identify individuals by tracking movement and posture. Trickier to disguise than a voice or face, harder to counterfeit than a written signature, such machine-readable kinematic watermarks challenge us to expand our conception of digital life-writing.

\section{ABOUT THE AUTHOR}

Rob Gallagher is a postdoctoral researcher with King's College London's EgoMedia project, which considers the role of digital technologies in fostering new forms of life narrative, modes of self-presentation and conceptions of identity. His research has appeared in journals such as Film Theory, Game Studies, Convergence and Games and Culture. He is the author of Videogames, Identity and Digital Subjectivity (Routledge, 2017). Email: robert.gallagher@kcl.ac.uk. 